Chronic Obstructive Pulmonary Diseases: Journal of the COPD Foundation

\author{
Original Research
}

\title{
Differences in Respiratory Symptoms and Lung Structure Between Hispanic and Non-Hispanic White Smokers: A Comparative Study
}

\author{
Alejandro A. Diaz, MD, MPH ${ }^{1}$ Farbod N. Rahaghi, MD, PhD ${ }^{1}$ Tracy J. Doyle, MD ${ }^{1}$ Thomas P. Young, BA ${ }^{1}$ \\ Erick S. Maclean, BS ${ }^{1}$ Carlos H. Martinez, MD $^{2}$ Raul San Jose Estepar, PhD $^{3}$ Stefano Guerra, MD $^{4}$ \\ Yohannes Tesfaigzi, $\mathrm{PhD}^{5}$ Ivan O. Rosas, $\mathrm{MD}^{1}$ George R. Washko, MD, $\mathrm{MSc}^{1}$ David O. Wilson, $\mathrm{MD}^{6}$
}

\section{Abstract}

Background: Prior studies have demonstrated that U.S. Hispanic smokers have a lower risk of decline in lung function and chronic obstructive pulmonary disease (COPD) compared with non-Hispanic whites (NHW). This suggests there might be racial-ethnic differences in susceptibility in cigarette smoke-induced respiratory symptoms, lung parenchymal destruction, and airway and vascular disease, as well as in extra-pulmonary manifestations of COPD. Therefore, we aimed to explore respiratory symptoms, lung function, and pulmonary and extra-pulmonary structural changes in Hispanic and NHW smokers.

Methods: We compared respiratory symptoms, lung function, and computed tomography (CT) measures of emphysema-like tissue, airway disease, the branching generation number (BGN) to reach a 2-mm-lumen-diameter airway, and vascular pruning as well as muscle and fat mass between 39 Hispanic and 39 sex-, age- and smoking exposure-matched NHW smokers.

Results: Hispanic smokers had higher odds of dyspnea than NHW after adjustment for COPD and asthma statuses (odds ratio[OR]=2.96; 95\% confidence interval [CI] 1.09-8.04), but no significant differences were found in lung function and CT measurements.

Conclusions: While lung function and CT measures of the lung structure were similar, dyspnea is reported more frequently by Hispanic than matched-NHW smokers.

\begin{abstract}
Abbreviations: chronic obstructive pulmonary disease, COPD; non-Hispanic white, NHW; computed tomography, CT; branching generation number, BGN; odds ratio, OR; confidence interval, CI; Pittsburgh Lung Screening Study, PLuSS; forced expiratory volume in 1 second, FEV $_{\mathbf{1}}$; forced vital capacity, FVC; forced expiratory flow between $25 \%$ and $75 \%$ of FVC, FEF $25 \%-\mathbf{7 5} \%$; percent of low attenuation areas below-950 Hounsfield units, \%LAA-950; right upper lobe apical bronchus, RB1; right lower lobe basal posterior bronchus, RB10; total blood vessel volume, TBV; pulmonary volume in all vessels $<10 \mathrm{~mm}^{\mathbf{2}}$ in cross section, BV10; pectoralis muscle area, PMA; subcutaneous adipose tissue, SAT; visceral adipose tissue, VAT; standard deviation, SD; body surface area, BSA

Funding Support: National Institutes of Health grant K01HL118714 and the Brigham and Women's Hospital Minority Faculty Career Development Award (Diaz).

Date of Acceptance: August 4, 2017

Citation: Diaz AA, Rahaghi FN, Doyle TJ, et al. Differences in respiratory symptoms and lung structure between Hispanic and non-Hispanic white smokers: A comparative study. Chronic Obstr Pulm Dis. 2017;4(4):297-304. doi: https://doi.org/10.15326/jcopdf.4.4.2017.0150
\end{abstract}

1 Division of Pulmonary and Critical Care Medicine, Brigham and Women's Hospital, Harvard Medical School, Boston, Massachusetts

2 Division of Pulmonary and Critical Care Medicine, University of Michigan Health System, Ann Arbor

3 Department of Radiology, Brigham and Women's Hospital, Harvard Medical School, Boston, Massachusetts
4 Asthma and Airway Disease Research Center and Department of Medicine, University of Arizona, Tucson; and ISGlobal CREAL and Pompeu Fabra University, Barcelona, Spain

5 Lovelace Respiratory Research Institute, Albuquerque, New Mexico

6 Division of Pulmonary, Allergy and Critical Care Medicine, University of Pittsburgh, Pittsburgh, Pennsylvania 


\section{Address correspondence to:}

Alejandro A. Diaz, MD, MPH

Division of Pulmonary and Critical Care Medicine

Department of Medicine

Brigham and Women's Hospital

75 Francis Street

Boston, MA 02115

Email: ADiaz6@Partners.org

\section{Keywords:}

chronic obstructive pulmonary disease; COPD; Hispanic/Latino; computed tomography; emphysema

\section{Introduction}

In 2015, 36.5 million people in the United States were current smokers. ${ }^{1}$ Before meeting criteria for chronic obstructive pulmonary disease (COPD), smokers manifest respiratory symptoms and have emphysematous destruction of lung parenchyma and airway disease that can be measured on computed tomography (CT) similar to those with established disease. ${ }^{2,3}$ Much of what we know about these clinical and radiographic manifestations in smokers comes from large studies that have included mostly nonHispanic white populations. ${ }^{2-5}$ Although Hispanics are the largest and fastest growing minority in the United States, ${ }^{6}$ now numbering 56.5 million people, and represent the majority population in certain areas of the United States, they are understudied. Interestingly, recent investigations suggest that despite comparable smoking habits and occupational exposures, Hispanic smokers (i.e., Hispanics from New Mexico and MexicanAmericans) have a slower rate of lung function decline and a lower risk for COPD than their non-Hispanic white (NHW) counterparts. ${ }^{7,8,9}$

These racial-ethnic differences in disease risk may be due to differential responses of the airways and lung parenchyma to tobacco injury. Detailed examination of lung structure and extra-pulmonary tissues would be necessary to show these differences. Prior studies did not find racial-ethnic differences in CT measures of emphysema and airway morphology. However, those studies were limited by their assessment only of one quantitative imaging phenotype (emphysema or airway disease) on cardiac CTs. ${ }^{10,11}$ Other CT phenotypes such as pulmonary vascular pruning and measures of extra-pulmonary tissues such as muscle and fat mass are now available, thereby expanding our ability to assess smoking-related diseases. ${ }^{12-15}$ In the present study, we aimed to explore pulmonary symptoms, lung function, and lung structure as well as muscle and fat tissue mass in Hispanic and NHW smokers. We tested the hypothesis that Hispanics might be less susceptible to smoking-induced injury compared to NHWs and that could be associated with greater airway lumens, more parallel pathways, and less emphysema and vascular pruning. To test this hypothesis, we used sex-, age-, and smoking exposure-matched Hispanic and nonHispanic whites from the Pittsburgh Lung Screening Study (PLuSS). ${ }^{16}$

\section{Methods}

\section{Cohort}

Clinical and CT data at baseline from participants enrolled in PLuSS from January 2001 to April 2005 were used. ${ }^{16}$ Inclusion criteria were the following: age 50-79 years; body weight $<400$ pounds; no personal history of lung cancer; nonparticipation in concomitant lung cancer screening studies; no chest CT in the prior year; former or current cigarette smoker of at least one-half pack/day for 25 or more years; former smokers must have quit within 10 years prior to study enrollment. Symptoms were not part of the exclusion criteria.

We used all self-identified Hispanic participants and matched them with non-Hispanic whites based on same sex, age ( \pm 2 years), and smoking exposure. Smoking exposure matching was based on the age when participants started smoking ( \pm 2 years) and quit smoking ( \pm 2 years) if applicable, the same current smoking status, and the same category of smoking intensity (cigarettes/day categories: 1-9; 10-19; 2029; 30-39; and 40 or more). This approach allowed us to explore potential differences in susceptibility to smoking-induced injury based on race-ethnicity. African-American individuals were not included in this study. The institutional review board for the University of Pittsburgh approved PLuSS and its participants gave informed consent to participate in the study. ${ }^{16}$ The informed consent was given to the participants in English only.

\section{Clinical and Functional Assessment}

Demographics, smoking history, and pulmonary symptoms (non-productive cough, phlegm, wheeze, and dyspnea) were collected in standardized manner using a PLuSS designed questionnaire, which was 
given to the participants in English only. Participants were asked, "Have you recently experienced any of the following symptoms?" The respiratory symptoms included "dry cough", "cough that produces phlegm", "wheezing", and "shortness of breath" with binary yes/ no response options. The participant was considered to have a respiratory symptom if he/she answered "yes" to any of those options. Spirometry was performed and analyzed following the American Thoracic Society standards. ${ }^{17}$ We used the forced expiratory volume in one second (FEV 1 ), forced vital capacity (FVC), FEV 1 / FVC ratio, and forced expiratory flow between $25 \%$ and $75 \%$ (FEF $25-75 \%$ ) of FVC and their predicted values in our analysis. The predicted values were derived from prediction equations for the U.S. population. ${ }^{18}$

\section{CT Protocol}

PLuSS participants were imaged using a singlebreath-hold, helical, low-dose CT protocol (40-60 mA; $140 \mathrm{kVp}$ ) to obtain axial images. The images were reconstructed with a high spatial frequency (lung) algorithm at contiguous 2.5-mm intervals. ${ }^{16}$

\section{Emphysema and Airway Assessment}

A trained analyst analyzed the CTs using the Chest Imaging Platform. ${ }^{19}$ Because PLuSS CTs are lowdose, images were first smoothed using a median filter allowing decreasing noise and increasing densitometric measurements accuracy. Emphysema-like tissue was defined as percent of low attenuation areas below -950 Hounsfield units (\%LAA-950). ${ }^{20}$ Measures of airway morphology were taken using an anatomic approach at the 3rd and 4th generation level of the right upper lobe apical bronchus (RB1) and right lower lobe basal posterior bronchus (RB10). The first RB1 and RB10 segment within the lobe was considered the third generation. ${ }^{21,22}$ RB 1 and R10 were chosen as they run orthogonal to the axial plane making it possible to take more accurate measurements. The analyst identified the target bronchial segment orthogonal to the axial image and placed a point at the center of the lumen to segment the airway and obtain the measurements. Two sets of bronchial measurements were obtained in the middle portion of each segment. These measurements included lumen area and wall area percentage (wall area/wall area + lumen area $\cdot 100) .{ }^{23}$ The ratio of the area of the daughter inner lumen to parent inner lumen was also computed and used to estimate the branching generation number (BGN) to reach a
$<2$ mm-lumen-diameter airway -the site of airflow obstruction in smokers as described previously. ${ }^{24}$ The $B G N$ is an estimation of how many branches (i.e., parallel pathways) there are between the proximal airways and a given distal airway size where greater values indicate greater number of parallel pathways. For this estimation, it is assumed that the bronchial tree is a dichotomous branching system where each generation had $2^{\mathrm{n}}$ parallel pathways (where " $\mathrm{n}$ " is the generation). If the projected BGN ranged from 6 to 8 (i.e., 3 to 5 generations past the 3 rd generation), then a participant would theoretically have between 64 and 256 parallel pathways of similar size (for a 6 th generation $2 \mathrm{~mm}$-airway $2^{6}=64$ parallel paths, for an 8 th generation $2 \mathrm{~mm}$-airway $2^{8}=256$ parallel paths)

\section{Pulmonary Vascular Assessment}

We used a 3-dimensional volumetric model to assess the pulmonary vascular morphology on non-contrast CT scans. ${ }^{15}$ This methodology allows extracting the vascular tree and quantifying the total blood vessel volume (TBV). This assessment provides the aggregate pulmonary vessel volume including artery and veins in all vessels less than $10 \mathrm{~mm}^{2}$ in cross section (BV10) for the whole-lung, a modified metric for low-dose CT scans from prior work. ${ }^{15}$ To account for differences in lung size across participants, the BV10 is expressed as a function of TBV. A lower BV10/TBV ratio indicates a greater vessel pruning.

\section{Muscle and Fat Assessment}

Measures of muscle and fat tissues were taken on chest $\mathrm{CT}$ scan. The methodology and reproducibility of these measures have been previously reported. ${ }^{12-14}$ We measured the pectoralis muscle area (PMA), ${ }^{12}$ subcutaneous adipose tissue $(\mathrm{SAT})^{13}$ area anterior to the pectoralis muscles, and visceral adipose tissue (VAT). ${ }^{14}$ The first 2 measurements are obtained from a single-axial slice immediately above the aortic arch and the latter is taken from a single-axial slice at the inferior edge of the transverse process of the first lumbar vertebrae. The aggregate area $\left(\mathrm{cm}^{2}\right)$ of the 4 pectoralis muscles and the 2 sides of SAT measurement as well as VAT total area were used for analysis.

\section{Statistical Analysis}

Data are presented as frequency (\%) or mean \pm standard deviation (SD). Differences between populations were tested using student t-tests or chi- 
squared tests for continuous and categorical variables, respectively. Logistic regression was performed to assess the relationship between a binary variable (i.e., presence of a respiratory symptom) and race-ethnicity. A $P$ value less than 0.05 was considered of statistical significance. The analysis was performed with SAS 9.4 (SAS institute, Cary, North Carolina) software package.

\section{Results}

Thirty-nine out of 3642 participants who underwent CT scanning at baseline were Hispanics. The Hispanic participants were successfully matched for age, sex, and smoking history with 39 NHWs. The participants' characteristics are shown in Table 1. Hispanic smokers had almost 2 times higher proportion of dyspnea

\section{Table 1. Characteristic of Selected Participants by Race/Ethnicity}

\begin{tabular}{|c|c|c|c|c|}
\hline \multirow{3}{*}{$\begin{array}{l}\text { Variable } \\
\text { Age, (yr) }\end{array}$} & \multirow{2}{*}{\multicolumn{2}{|c|}{$\begin{array}{c}\text { NHW } \\
(\mathrm{N}=39) \\
\text { Mean } \pm \\
\text { SD or \% }\end{array}$}} & \multirow{2}{*}{\multicolumn{2}{|c|}{$\begin{array}{l}\text { Hispanics } \\
(\mathrm{N}=39) \\
\text { Mean } \pm \\
\text { SD or } \%\end{array}$}} \\
\hline & & & & \\
\hline & 58 & \pm 5 & 57 & \pm 5 \\
\hline Male Sex, \% & 41 & & 41 & \\
\hline Height, (m) & 1.68 & \pm 0.09 & 1.65 & \pm 0.1 \\
\hline Weight, (kg) & 80.8 & \pm 20.0 & 79.9 & \pm 15.1 \\
\hline Smoking > 20 cigarettes/day, \% & 56 & & 56 & \\
\hline Current smoking status, $\%$ & 72 & & 72 & \\
\hline Age Start Smoking, (yr) & 17 & \pm 3 & 17 & \pm 3 \\
\hline Age Stop Smoking, (yr) & 53 & \pm 7 & 53 & \pm 7 \\
\hline Smoking Duration, (yr) & 39 & \pm 7 & 39 & \pm 7 \\
\hline Cough, \% & 49 & & 41 & \\
\hline Phlegm, \% & 64 & & 51 & \\
\hline Wheeze, \% & 36 & & 54 & \\
\hline Dyspnea $^{a}, \%$ & 33 & & 65 & \\
\hline History of Asthma, \% & 10 & & 23 & \\
\hline Spirometric COPD, \% & 46 & & 46 & \\
\hline $\mathrm{FEV}_{1},(\mathrm{~L})$ & 2.5 & \pm 0.7 & 2.3 & \pm 0.8 \\
\hline $\mathrm{FEV}_{1},(\%$ predicted $)$ & 83 & \pm 17 & 81 & \pm 20 \\
\hline FVC, (L) & 3.6 & \pm 0.9 & 3.3 & \pm 1.1 \\
\hline FVC, $(\%$ predicted $)$ & 91 & \pm 15 & 89 & \pm 16 \\
\hline $\mathrm{FEV}_{1} / \mathrm{FVC},(\%)$ & 70 & \pm 10 & 70 & \pm 13 \\
\hline FEF 25-75, (L) & 1.8 & \pm 0.9 & 1.8 & \pm 1.2 \\
\hline FEF $25-75,(\%$ predicted $)$ & 64 & \pm 30 & 67 & \pm 42 \\
\hline
\end{tabular}

Data are presented as proportion (\%) for categorical variables or mean \pm standard deviation (SD) for continuous variables.

${ }^{\mathrm{a}} P<0.05$.

Absence of "a" indicates $P \geq 0.05$ than their NHW counterparts. The increased odds of dyspnea for Hispanics persisted after adjustment for both spirometrically defined COPD and history of asthma (odds ratio[OR]=2.96; 95\%, confidence interval [CI] 1.09-8.04). No significant differences were observed in other respiratory symptoms, FEV $\mathrm{F}_{1}$, FVC, and $\mathrm{FEV}_{1} / \mathrm{FVC}$.

\section{CT Measurements}

No significant differences were found in any of the CT measurements (airway morphology, BGN, \%LAA950, vascular morphology, PMA, SAT, and VAT) between Hispanics and NHWs (Table 2). The lack of racial-ethnic differences in airway lumen size was also observed when RB1 airway lumen area was adjusted for body surface area (BSA) as lumen area/BSA or height as lumen area $/ \mathrm{m}^{2}$ ( $P>0.73$ for both comparisons).

\section{Discussion}

In this study, we performed an evaluation of respiratory symptoms and lung function as well as a comprehensive analysis of CT lung measurements and extra-pulmonary tissues from Hispanic and matched-NHW smokers. We found that Hispanics reported almost two-fold more dyspnea than NHWs. However, racial-ethnic differences in lung function, lung structure, and extra-pulmonary tissues were not observed.

Smokers with and without COPD frequently report dyspnea. In our study, despite Hispanics and NHWs having similar CT-based lung structural damage and lung function, the minority group reported almost twice more often dyspnea. An explanation for this difference is that compared to NHWs with similar lung function, Hispanic smokers may have a lower perception threshold for this symptom. We have previously reported that New Mexican Hispanic smokers (versus NHWs) more often reported dyspnea in univariate analysis, and that they also experience a worse perception of their healthrelated quality of life as measured by the St George's Respiratory Questionnaire that includes questions about this symptom. ${ }^{25}$ Additionally, the feeling of "breathlessness" is described with both upper airway (e.g., tight throat) and lower airway word descriptors (e.g., sore lung) by Hispanics compared to just the lower airway word descriptors used by $\mathrm{NHWs}^{26}$ 


\section{Table 2. CT Measurements of Participants by Race/Ethnicity}

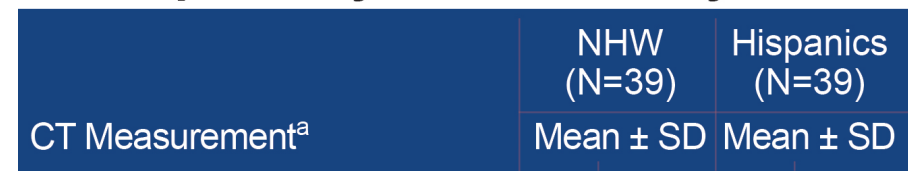

\begin{tabular}{|c|c|c|c|}
\hline \multicolumn{4}{|c|}{ Airway Lumen Area $\left(\mathrm{mm}^{2}\right)$} \\
\hline RB1 Generation $3^{\text {rd }}$ & 20.3 & \pm 6.5 & $20.1 \pm 9.2$ \\
\hline RB1 Generation $4^{\text {th }}$ & 10.5 & \pm 4.0 & $10.9 \pm 4.9$ \\
\hline RB10 Generation $3^{\text {rd }}$ & 22.3 & \pm 7.8 & $22.4 \pm 8.0$ \\
\hline RB10 Generation $4^{\text {th }}$ & 14.1 & \pm 5.6 & $14.8 \pm 6.7$ \\
\hline \multicolumn{4}{|l|}{ Wall Area Percent (\%) } \\
\hline RB1 Generation $3^{\text {rd }}$ & 51.5 & \pm 5.2 & $52.3 \pm 7.1$ \\
\hline RB1 Generation $4^{\text {th }}$ & 59.2 & \pm 7.6 & $59.7 \pm 7.8$ \\
\hline RB10 Generation $3^{\text {rd }}$ & 50.7 & \pm 5.8 & $50.9 \pm 5.6$ \\
\hline RB10 Generation $4^{\text {th }}$ & 56.7 & \pm 6.8 & $57.2 \pm 8.1$ \\
\hline
\end{tabular}

\begin{tabular}{l|c|c|c|c|}
\hline Branching Generation Number $^{b}$ & 6.8 & \pm 2.4 & 7.3 & \pm 2.9
\end{tabular}

(No. of generations)

\begin{tabular}{l|r|r|r|r}
\hline LAA-950 (\%) & 3.6 & \pm 6.9 & 2.7 & \pm 4.8 \\
\hline Vascular Morphology & & & & \\
\hline BV10, ml & 101 & \pm 23 & 101 & \pm 24 \\
\hline TBV, ml & 186 & \pm 41 & 184 & \pm 39 \\
\hline BV10/TBV & 0.54 & \pm 0.06 & 0.55 & \pm 0.05 \\
\hline Muscle and Adipose Tissue $\left(\mathrm{cm}^{2}\right)$ & & & & \\
\hline PMA & 36 & \pm 13 & 38 & \pm 14 \\
\hline SAT & 39 & \pm 17 & 42 & \pm 17 \\
\hline VAT & 164 & \pm 52 & 167 & \pm 46 \\
\hline Total Lung Volume $(\mathrm{L})$ & 5.3 & \pm 1.2 & 5.1 & \pm 1.5 \\
\hline
\end{tabular}

${ }^{a} P>0.05$ for the difference in each measurement listed.

${ }^{b}$ Branching generation number to reach $\mathrm{a}<2-\mathrm{mm}$-lumen-diameter airway

LAA-950\%=low attenuation areas below -950 Hounsfield units; RB1=right upper lobe apical bronchus; RB10=right lower lobe basal posterior bronchus; BV10=pulmonary vessel volume in vessels less than $10 \mathrm{~mm}^{2}$ in cross section; TBV=total blood vessel volume; $\mathrm{PMA}=$ pectoralis muscle area; $\mathrm{SAT}=$ subcutaneous adipose tissue; $\mathrm{VAT}=$ abdominal visceral adipose tissue.

supporting the racial-ethnic difference we observed. But an additional prior study in Mexican-Americans did not find differences in this symptom compared to NHWs. ${ }^{7}$ That study, however, included never smokers, which may have obscured the differences we observed in heavy smokers. A potential implication of current and prior findings is that these racial-ethnic differences in dyspnea perception might be considered when evaluating a Hispanic smoker in routine clinical visits.

We also performed a comprehensive chest CT assessment to explore potential Hispanic-NHW structural differences in response to tobacco injury. We were surprised by both the remarkable similarity in some of the imaging estimates (e.g., airway lumen area, vascular pruning) and the consistent lack of differences in all structures/tissues evaluated between the 2 populations. One possible explanation of our findings is that racial-ethnic differences in tobaccoinduced injury do not translate into morphologic responses of lung structure. Other studies have failed to find racial-ethnic differences in emphysema and airway disease ${ }^{10,11}$ and here the lack of difference was expanded to vascular pruning and extra-pulmonary manifestations of COPD such as muscle and fat mass. Although some may feel that our sample size might obscure potential differences, the close estimates for some CT measurements between the populations make this less likely. However, these findings do not exclude the possibility that differences in smokinginduced changes of lung structures might exist between other Hispanic subgroups and NHWs. For example, a cohort of Hispanics from New Mexico with greater Native American ancestry than that of NHWs had lower risk of COPD. ${ }^{8}$ This suggests that lung structure might differ by ancestry rather than ethnicity as a broad category. Additionally, differences in other structures that were not measured in our study such as small airways may exist. ${ }^{27}$ Furthermore, differences in inflammatory response to smoking injury, and/or protease-anti-protease misbalance may explain racialethnic differences in COPD risk previously observed. Further investigations including imaging, genetics, and environmental assessments are warranted to elucidate the determinants of COPD risk and disease progression in all U.S. racial-ethnic groups.

Several limitations should be noted. First, this is a convenience, small-sized sample of heavy smokers and caution should be exercised to extrapolate these findings to the larger population of smokers or Hispanics as a whole. We do not have information on ancestry (European, Native American, African), nativity (U.S. born versus non-U.S. born), and origin (e.g., Mexican-American, Puerto Ricans) of these participants, thus those important characteristics that are associated with observed differences in lung function decline and COPD risk could not be factored into our analysis. ${ }^{8,28}$ While this lack of information limits the external validity of our findings, it might encourage further exploration of smoking-induced changes in lung structure and COPD susceptibility based on race-ethnicity using more refined methods to characterize populations such as ancestry. The 
assessment of respiratory symptoms was based on their presence or absence only and smoking intensity was collected as category of cigarettes/day. Finally, COPD measures on expiratory images such as air trapping were not available. Despite this limitation, we were able to expand prior imaging characterization in Hispanics by analyzing the pulmonary vasculature and extra-pulmonary tissues. Low-dose CT scans imposed challenges for lung densitometric analysis due to a reduced signal/noise ratio, but smoothing the PLuSS images allowed us to obtain emphysema-like tissue measurements that were in the range of those reported from studies using high-dose CT scans.

In summary, Hispanic heavy smokers report dyspnea 2 times more often than their age-, sex- and smoking exposure-matched NHW counterparts. It appears that there are no differences in CT measures of COPD between Hispanic and NHW smokers. Further research including imaging, genetics, and environmental exposure assessments are needed to ascertain the determinants of COPD and disease progression in all U.S. racial-ethnic groups.

\section{Acknowledgments}

Authors' Contributions: Conception and design of this study and creation, revision, and final approval of this manuscript: Authors Diaz, Rahaghi, Doyle, Young, Maclean, Martinez, Jose Estepar, Guerra, Tesfaigzi, Rosas, Washko and Wilson; Analysis and interpretation: Diaz, Washko, Wilson; Data acquisition: Diaz, Rahaghi, Young, Wilson; Drafting the manuscript for important intellectual content: Diaz, Rahaghi, Doyle, Guerra, Tesfaigzi, Wilson; Guarantor Diaz had full access to all of the data in the study and takes responsibility for the integrity of the data and the accuracy of the data analysis.

\section{Declaration of Interest}

Drs. Diaz, Rahaghi, Doyle, Martinez, San Jose Estepar, Guerra, Tesfaigzi, Rosas and Wilson, and Mr. Young and Mr. Maclean have no conflicts of interest to disclose related to this manuscript. Dr. Diaz has received speaker fees unrelated to this work from Novartis, Inc. 


\section{References}

1. Jamal A, King BA, Neff LJ, Whitmill J, Babb SD, Graffunder CM. Current cigarette smoking among adults - United States, 20052015. MMWR Morb Mortal Wkly Rep. 2016;65(44):1205-1211. doi: https://doi.org/10.15585/mmwr.mm6544a2

2. Regan EA, Lynch DA, Curran-Everett D, et al. Clinical and radiologic disease in smokers with normal spirometry. JAMA Intern Med. 2015;175(9):1539-1549.

doi: https://doi.org/10.1001/jamainternmed.2015.2735

3. Woodruff PG, Barr RG, Bleecker E, et al. Clinical significance of symptoms in smokers with preserved pulmonary function. New Eng J Med. 2016;374(19):1811-1821.

doi: https://doi.org/10.1056/NEJMoa1505971

4. Washko GR, Diaz AA, Kim V, et al. Computed tomographic measures of airway morphology in smokers and never-smoking normals. J App Physio. 2014;116(6):668-673.

doi: https://doi.org/10.1152/japplphysiol.00004.2013

5. Hansel NN, Washko GR, Foreman MG, et al. Racial differences in CT phenotypes in COPD. COPD. 2013;10(1):20-27. doi:https://doi.org/10.3109/15412555.2012.727921

6. Barr RG, Aviles-Santa L, Davis SM, et al. Pulmonary disease and age at immigration among Hispanics. Results from the Hispanic Community Health Study/Study of Latinos. Am J Respir Crit Care Med. 2016;193(4):386-395.

doi: https://doi.org/10.1164/rccm.201506-12110C

7. Diaz AA, Come CE, Mannino DM, et al. Obstructive lung disease in Mexican Americans and non-Hispanic whites: An analysis of diagnosis and survival in the NHANES III follow-up study. Chest. 2013;145: 282-289.

doi: https://doi.org/10.1378/chest.13-1414

8. Bruse S, Sood A, Petersen H, et al. New Mexican Hispanic smokers have lower odds of chronic obstructive pulmonary disease and less decline in lung function than non Hispanic whites. Am J Respir Crit Care Med. 2011;184(11):1254-1260. doi: https://doi.org/10.1164/rccm.201103-05680C

9. Petersen H, Sood A, Meek PM, et al. Rapid lung function decline in smokers is a risk factor for COPD and is attenuated by angiotensin-converting enzyme inhibitor use. Chest. 2014;145(4):695-703. doi: https://doi.org/10.1378/chest.13-0799

10. Powell R, Davidson D, Divers J, et al. Genetic ancestry and the relationship of cigarette smoking to lung function and per cent emphysema in four race/ethnic groups: a cross-sectional study. Thorax. 2013;68(7):634-642.

doi: https://doi.org/10.1136/thoraxjnl-2012-202116

11. Donohue KM, Hoffman EA, Baumhauer H, et al. Cigarette smoking and airway wall thickness on CT scan in a multi-ethnic cohort: the MESA Lung Study. Respir Med. 2012;106(12):16551664. doi:https://doi.org/10.1016/j.rmed.2012.08.006
12. McDonald ML, Diaz AA, Ross JC, et al. Quantitative computed tomography measures of pectoralis muscle area and disease severity in chronic obstructive pulmonary disease. A crosssectional study. Annals Am Thorac Soc. 2014;11(3):326-334. doi: https://doi.org/10.1513/AnnalsATS.201307-2290C

13. Diaz AA, Zhou L, Young TP, et al. Chest CT measures of muscle and adipose tissue in COPD: gender-based differences in content and in relationships with blood biomarkers. Acad Radiol. 2014;21(10):1255-1261. doi: https://doi.org/10.1016/j.acra.2014.05.013

14. Diaz AA, Young TP, Kurugol S, et al. Abdominal visceral adipose tissue is associated with myocardial infarction in patients with COPD. Chronic Obstr Pulm Dis. 2015;2(1):8-16. doi: https://doi.org/10.15326/jcopdf.2.1.2015.0127

15. Estepar RS, Kinney GL, Black-Shinn JL, et al. Computed tomographic measures of pulmonary vascular morphology in smokers and their clinical implications. Am J Respir Crit Care Med. 2013;188(2):231-239.

doi: https://doi.org/10.1164/rccm.201301-0162OC

16. Wilson DO, Weissfeld JL, Fuhrman CR, et al. The Pittsburgh Lung Screening Study (PLuSS): outcomes within 3 years of a first computed tomography scan. Am J Res Crit Care Med. 2008; 178(9):956-961.

doi: https://doi.org/10.1164/rccm.200802-3360C

17. American Thoracic Society. Standardization of spirometry, 1994 update. Am J Respir Crit Care Med. 1995;152(3):1107-1136. doi: https://doi.org/10.1164/ajrccm.152.3.7663792

18. Hankinson JL, Odencrantz JR, Fedan KB. Spirometric reference values from a sample of the general U.S. population. Am J Respir Crit Care Med. 1999;159(1):179-187. doi: https://doi.org/10.1164/ajrccm.159.1.9712108

19. Harvard University. Chest imaging platform. Chest Imaging website https://www.chestimagingplatform.org. Accessed July 2017.

20. Gevenois PA, De Vuyst P, de Maertelaer V, et al. Comparison of computed density and microscopic morphometry in pulmonary emphysema. Am J Respir Crit Care Med. 1996;154(1):187-192. doi: https://doi.org/10.1164/ajrccm.154.1.8680679

21. Diaz AA, Valim C, Yamashiro T, et al. Airway count and emphysema assessed by chest CT imaging predicts clinical outcome in smokers. Chest. 2010;138(4):880-887. doi: https://doi.org/10.1378/chest.10-0542

22. Yamashiro T, Matsuoka S, Estepar RS, et al. Quantitative airway assessment on computed tomography in patients with alpha1antitrypsin deficiency. COPD. 2009;6(6):468-477. doi: https://doi.org/10.3109/15412550903341521

23. Diaz AA, Han MK, Come CE, et al. Effect of emphysema on CT scan measures of airway dimensions in smokers. Chest. 2013;143(3):687-693. doi: https://doi.org/10.1378/chest.12-0039 
24. Diaz AA, Rahaghi FN, Ross JC, et al. Understanding the contribution of native tracheobronchial structure to lung function: CT assessment of airway morphology in never smokers. Respir Res. 2015;16:23.

doi: https://doi.org/10.1186/s12931-015-0181-y

25. Diaz AA, Petersen H, Meek P, Sood A, Celli B, Tesfaigzi Y. Differences in health related quality of life between New Mexican Hispanic and non-Hispanic white smokers. Chest. 2016;150(4):869-876.

doi: https://doi.org/10.1016/j.chest.2016.06.011

26. Hardie G, Liu R, Darden J, Gold WM. Ethnic differences in methacholine responsiveness and word descriptors in African Americans, Hispanic-Mexican Americans, AsianPacific Islanders, and whites with mild asthma. J Asthma. 2010;47(4):388-396.

doi: https://doi.org/10.3109/02770903.2010.481341

27. Bhatt SP, Soler X, Wang X, et al. Association between functional small airway disease and FEV1 decline in chronic obstructive pulmonary disease. Am J Respir Crit Care Med. 2016;194(2):178184.

doi: https://doi.org/10.1164/rccm.201511-22190C

28. Chen W, Brehm JM, Boutaoui N, et al. Native American ancestry, lung function, and COPD in Costa Ricans. Chest. 2014;145(4):704-710.

doi: https://doi.org/10.1378/chest.13-1308 